\title{
TEKHNOPAIGNION QUE APARECE EN LOS ESCOLIOS DE ILÍADA Z. 87-89. UNA INTERPRETACION.
}

\author{
ROSANA LEPORE \\ Universidade de Buenos Aires
}

Resumo: A comparaçăo entre os versos 86-89 / 269-270 da lliada Z, um fragmento dos Comentarii ad Homeri liadem de Eustácio e o texto do technopaegnium estudado permitem-nos fazer uma interpretaçáo oposta à dos filologos que consideram que o escólio é construldo como poema icónico. Por outro lado, o exame do technopaegnium ressalta sua função e a articulaçáo da forma e do conteúdo no interior do poema. A conclusáo do trabalho deriva da análise do nivel sintático e semántico no technopaegnium e dos hexâmetros em questăo.

Palavras-chave: Homero, poesia grega, escolios a Homero, tecnopalgnion.

A partir de nuestra interpretación del tekhnopalgnion que, como sabemos, es un escolio de lliada $Z, 87-89$, pretendemos demonstrar, em primer lugar, cual es el motivo que ha despertado el interés del escoliasta por dar una explicación a la estructura sémica de estos hexámetros. Dicha estructura ofrece dificultades de comprensión si nos atenemos al plano sintáctico. En segundo lugar, intentaremos realizar un análisis del tekhnopaignion teniendo en cuenta la relación contenido-forma. (Ver desenho pág. 14)

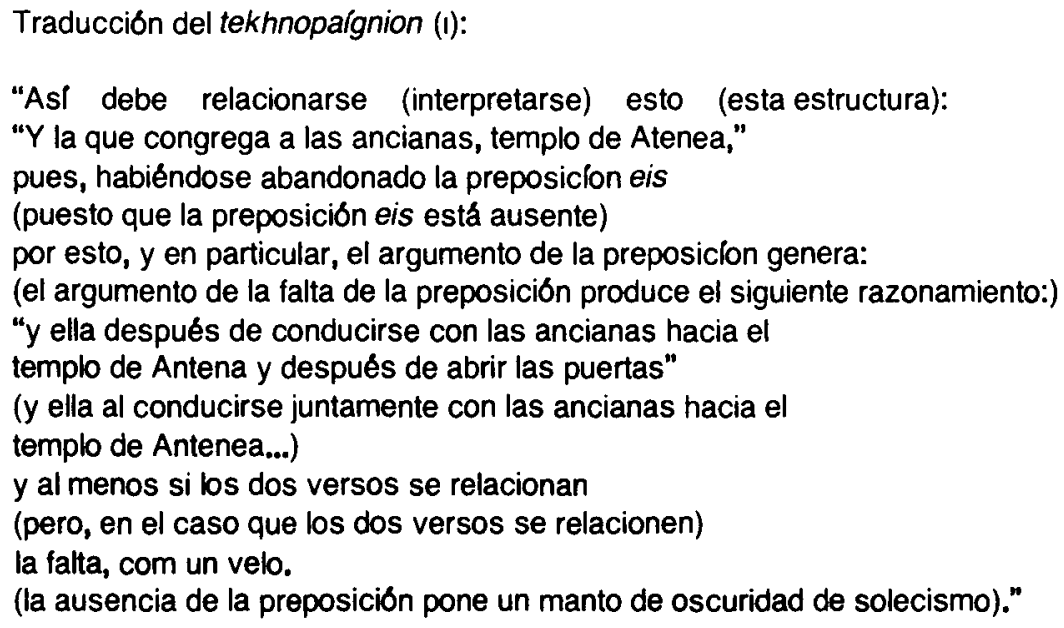

(1) Lo que no se halla entre parentesis es traducción literal. Las formas apocopadas, la concentración y la paratáxis de las freses dificultan la traducción del tekhnopalgnion, además de revelar que el interés del escoliasta está puesto en la observación fí lológica y en la concreción formal, en la disposición de su propuesta a modo de caligrama. 


$$
\begin{aligned}
& \Delta \quad 0 \quad \ddot{v} \Delta \\
& \tau \omega
\end{aligned}
$$

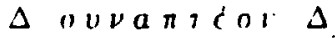

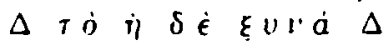

$$
\begin{aligned}
& \gamma \circ u \\
& \text { o } a \\
& \gamma \epsilon \\
& \rho a l \\
& \text { à } \varsigma \\
& \tau \omega \\
& \nu \eta \\
& \Delta \circ v a \Delta
\end{aligned}
$$

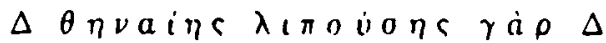

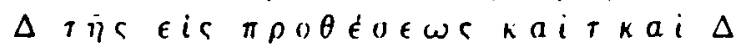

$$
\begin{aligned}
& \Delta \circ \cup \mu \Delta \\
& \delta \in C \\
& \mu \circ v \\
& \gamma \quad i \\
& \nu \quad E \\
& \text { Tat }
\end{aligned}
$$$$
\delta \lambda \dot{0}
$$$$
\gamma \circ \mathrm{c}
$$$$
\eta \delta \dot{\epsilon}
$$$$
\xi \cup v \dot{a}
$$$$
\text { rou }
$$$$
\text { o } a
$$$$
\text { tais }
$$$$
\text { repai }
$$$$
\text { a is }
$$$$
\text { E ic }
$$$$
\tau \dot{0} \nu
$$

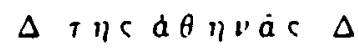

$$
\text { vad kai }
$$$$
\dot{a} \nu 0 i \xi a \cup a
$$$$
\text { Tás } \theta \text { lipas } \dot{\epsilon}
$$

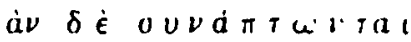

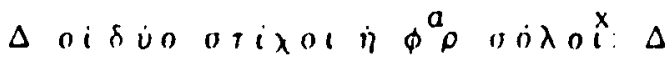


Algumas estructuras sintácticas de la poesia de Homero nos iofrecen ı más de una posibilidad de lectura; la polisemia del discurso y la permanente remisión a lo implicito nos conducen, mediante la síntesis de la expresión y la reducción de la realidad en la palabra exacta, a la bien tramada estructura profunda en la que el juego de "lo no dicho" es la clara apelación del poeta a la potencialidad intelectiva del hombre. Por esta razón, es posible una lectura capaz de rebatir la solución propuesta por el escoliasta respecto del problema sintáctico y morfológico que es expuesto e interpretado en el tekhnopaignion. A continuacion pasaremos a desarrollar una solución que creemos admisible a partir de la interpretacion del escoliasta.

En este tekhnopargnion se pretende dilucidar la forma en que deben ser relacionados e interpretados (hoúto synaptéon tò: he dè xynágousa geraiás...) los siguientes hexámetros, que corresponden a un parlamento de Heleno ${ }^{2}$ Illíada Z, 87-89:

"... he dè xynágousa geraiás

neòn Athenales glaukópidos en pólei ákrei,

olxasa kleîdi thyras hieroío dómoio,"

El escoliasta respecto de la construcción "nêón Athenaies glaukópidos" argumenta "... lipoúses gàr tês eis prothéseos..." En consecuencia reduce el conflicto conceptual a la falta de la preposición eis y propone, mediante la reposicion de un elemento gramatical, la siguiente interpretación del sintagma:

"... hê dè xynágousa taîs geraiaîs eis tòn tês Athenâs naòn kai anoíxasa tàs thyras..."

De esta manera, por medio de la reposición de eis resuelve un poî dependiente del participio xynágousa, y obtiene con el dativo taîs geraiaîs un régimen del preverbio syn-; de ese modo, el poî pasa a modificar plenamente al verbo ágo que ya no carga con el matiz semántico que le otorga el preverbio, puesto que, en esas condiciones, syn-forma con el dativo taîs geraiaîs una construcción independente que modifica a agó en su calidad de verbo de movimiento. Mediante el coordinante copulativo kaí los dos participios xynágousa y anoíxasa quedan en el mismo nivel sintáctico, y, por esta razón, el participio xynágousa pierde un tanto su valencia de sustantivo al mismo tiempo que se reviste de un valor pronominal.

Luego, el escoliasta añade: "... eàn dè synáptontai hoi dúo stikhoi, hé phar sóloi". Si bien se comprende la idea de movimiento, de peregrinación hacia el templo de Atenea, como una secuencia de acciones, el creador del tekhnopalgnion cierra su obra con una duda en cuanto a la relación semántica de estos hexámetros, y la manifiesta con una proposición condicional eventual que practicamente linda con una concesión, dejando abierta la posibilidad de una falta de claridad o de un solecismo.

Al confrontar este parlamento de Heleno con el de Héctor (II. Z, 263 ss), podemos advertir ciertas variantes; éstas, tal vez, pudieron haber influido en la interpretación del escoliasta respecto de II. Z, 87-89. Si bien los siguientes hexámetros fundamentan su interpretación por algunas razones, por otras la contrarrestan: "allà sy mèn pròs neón Athenaíes agaleles / érkheo syn thyeesin, aollissasa geraiás" (II. Z, 269-270). Aqui la idea de movimiento poî, que depende de érkhomai, es indiscutible y conceptualmente se aproxima a la propuesta de reposición desarrollada en el tekhnopalgnion; pero hay un lexema que desmoronarla la propuesta del escolio, éste es el participio aollissasa que, en posición quiásmica respecto de sy, funciona más en una re-

(2) Frente a las bajas troyanas provocadas por la acción bélica de Diomedes, y las incursiones del plano divino en el campo de batalta, Heleno aconseja a Hector que se dirija a la ciudad para pedirle a Hecuba que junto con las ancianas invoque a Atenea en la Acrópolis. 
lación apositiva que sustancialmente modifica al pronombre personal sy - casi como eplteto-, que en una relación apositiva implicando una acción meramente puntual.

Como podemos observar, estos dos hexámetros conforman una estructura quiásmica perfecta:

lliada Z, 270 / lliada Z, 269

érkheo syn thyeesin / prós neón Athenaies ageleles,

y: que apunta a una secuencia de movimiento puntal y precisa,

Iliada Z 269 / lliada Z, 270

allà sy mèn / aollissasa geraiás,

que pone de relieve a Hécuba en su calidad de basileia, en esas circunstancias, siempre aollíssasa geraiás y siempre xynágousa geraiás.

Por este motivo no estamos de acuerdo con la interpretación que nos ofrece el tekhnopaígnion, porque creemos que el participio xynágousa del v. 87 está sustantivado, y que conforma con el acusativo geraiás una unidad semántica en la que el preverbio syn- pierde su valor prepositivo y recobra su originario valor adverbial que modifica sustancialmente al verbo ágo.

El escoliasta, al darle una valencia pronominal al artículo - que, para nosotros, seria la marca de sustantivación del participio - trata de desmembrar el núcleo sémico hé dè xynágousa geraiás para otorgarle valencia preposicional al syn- que requiere la presencia del dativo taís geraiaîs. De esta manera, una vez resuelto el problema que le origina el preverbio, obtiene en el verbo àgo la idea de movimento que justifica la presencia del poî: (eis) neón Athenales y la reposición de la preposición eis, aproximándose, asf, a la secuencia de acción de II. Z. 269-270 (parlamento de Hector).

Eustacio, em Comentarii ad Homeri lliadem, manifesta: eipòn goûn Hellenos neơn Athenaies epágei: olxasa kleîdi thýras hieroío dómoio". En este pasaje, nedn Athenales depende del preverbio epl que incide en ágo sin modificar la idea sustancial de movimiento de este verbo. La interpretación de Eustacio no difiere de aquella que propone el tekhnopalgnion y que con claridad se manifiesta en II. Z, 269-270. Cómo explicarnos, entonces, la presencia de la construcción neón Athenales, núcleo sémico del discurso en el cual está inserta -que, por lo que hemos demostrado, no depende del participio xynágousa dado que el preverbio modifica el valor de ágo como posible verbo de movimento poî y, a su vez, lo vincula al acusativo geraiás, conformando una unidade semántica que apunta a Hecuba en su calidade de basilela; tampoco es posible hacer depender a neòn Athenales del participio olxasa del hexámetro siguiente puesto que éste forma parte de la secuencia de acción ligada a kleidi thýras hieroío dómoio.

Si bien la construcción neơn Athenales está ubicada en inicio de hexámetro, desmembrada, en aparencia, del resto del sintagma y lindando con um poú: en pólei ákrei, ubicado en final de hexámetro, la construcción neòn Athenales glaukopidos se encuentra en una posición de lectura intermedia dentro de una estructura en tres: se halla en relación quiásmica con he dè xynágousa geraiás que, a su vez, conforma una unidad de sentido con el poû: en pólei ákrei del hexámetro siguiente.

Por lo tanto, sin pensar en reducir la cuestión a una exigencia métrica y sin pensar en una falta de claridad, como un velo que opaca el mensaje de estos versos, sino, basándonos en la sintesis semántica y en la concentración expresiva de la poesia de Homero, creemos que esa posición de neòn Athenaíes sugiere la intención del poeta de dar relevancia a este núcleo sémico, que, desmembrado, en aparencia, de los lexemas lindantes por su ubicación en el hexámetro, los atrae sobre si, los carga de significado y los vincula. Creemos que a través de la construcción neòn Athenaies se apunta a partir de lo concreto a la abstracción o a la metáfora de la pólis en desgracia, dentro de un contexto de advertencia en el cual la pólis con su des- 
trucción y su dolor debe ser, mediante la invocación a Atenea, el punto de confluencia y de reencuentro de la divinidad y el hombre.

Por este motivo, vemos en la estructura neòn Athenafes un acusativo pleno, prácticamente absoluto, que modifica al poû: en pólei ákrei, como aposición del mismo, y que sintetiza el sintagma he dè xynágousa geraiás es decir, he dè xynágousa geraiás en pólei ákrei es neơn Athenaies glaukopidos. Pensamos, tambien que al desplegar la construcción neón Athenaies, que funciona como aposición de dos núcleos sémicos, conformando con ellos una unidad, emerge el siguiente concepto: es necesario reunirse en la Acrópolis que es templo de Atenea, y es necesario ser el templo de Atenea, la arquitectura humana del ruego y de la ofrenda. De la ásty condenada a la destrucción debe erguirse he dè xynágousa geraiás, Hécuba, he basileia: la madre con las madres, la pólis suplicante, para darle justificación divina y humana al dómos hierós.

Hemos podido comprobar que el escoliasta fundamenta su razonamiento filológico en la ausencia de la preposición eis: lipoúses gàr tês eis prothéseos. Y formula -mediante la reposición de este elemento lexical- la interpretación que es desplegada en el centro del tekhnopaígnion:

“... he dè xynágousa taîs geraiaís eis tòn tês Athenâs naòn kai anolxasa tàs thýras...",

de la que se desprende una conclusión:

"... eản dè synáptontai hoi dúo stkkhoi, he phar sóloi:"

que insinúa una falta de claridad semántica en la estructura sintáctica de los hexámetros 87-89 de II. Z:

"... he dè xynágousa geraiás

neòn Athenaies glaukópidos en pólei ákrei,

oíxasa kleîde thyras hieroî dómoio,"

A partir de la propuesta desarrollada en el escolio, hemos formulado una interpretación que difiere de aquella expuesta en el tekhnopaígnion, limitándonos, en primer lugar, a las normas sintácticas establecidas, y en segundo lugar, basándonos en la sintesis expresiva del lenguaje poético.

Por lo demostrado, podemos afirmar que esta especie de acrobacia artistica o juego literario es una creación absolutamente funcional; dado que se trata de un escolio, no nos es posible emitir un juicio de valoración estética respecto de su contenido fuera del ámbito especifico al cual pertence. Es en el particular e ingenioso continente donde, creemos, radica la importancia y el mérito creativo de la obra.

El escoliasta dispone su concisa argumentación a modo de caligrama y somete a una forma determinada la riqueza conceptual y la claridad de su interpretación. Cabe preguntarse, entonces, cuál es la relación entre la disposición formal de su discurso y el objeto que éste pretende designar.

En primer lugar, existe la posibilidad de pensar que el tekhnopalgnion configure un templo, al asociar este referente con el signo neòn -lexema que genera dificultades de comprensión y la necessidad del escoliasta de despejar una estructura compleja. En segundo lugar, existe la posibilidad de pensar que el tekhnopaígnion representa una llave; consideramos que este último argumento es el más acertado por la conclusión que se desprende del seguiente análisis.

El dativo instrumental kleíde, que modifica al participio ofxasa del hexámetro 89 de $11 . \mathrm{Z}$, se halla en relación quiásmica con el acusativo neòn del hexámetro 88; si bien ambos lexemas 
están semánticamente vinculados, cada uno de estos forma parte de una unidad sémica bien diferenciada por el plano sintáctico: la estructura he dè xynágousa geraiás neòn Athenaies glaukópidos en pólei ákrei, es independiente de la estructura oíxasa kleîdi thyras hieroio dómoio, aunque en el plano sémico volvamos a estar en presencia de una reconcentrada interacción de todos los constituyentes del sintagma.

Oíxasa kleîdi thýras hieroî dómoio, conforma una dinámica secuencia de acción, por lo tanto resulta notable el hecho de que el escoliasta no haya reparado en el lexema kleide y lo haya evitado en la reproducción del hexámetro 89 de 1 . Z, que intenta transcribir en el centro del tekhnopaígnion. Esto nos permite inferir que formalmente la obra, el escolio, es la réplica de una llave.

De este modo, el creador apela, como Homero, a la capacidad intelectiva del hombre, evitando plasmar la palabra, que, por una parte, encierra y enmarca, enigmáticamente, el contenido del escolio -mimetizando la forma del referente mediante la ausencia expresa' del signoy que, por otra parte, abre y despliega, a modo de caligrama, una lectura que supone esclarecedora.

Creemos que el escoliasta, mediante un tekhnopaignion, intenta reproducir la dificultad que le ocasiona la ausencia de la preposición eis con la ausencia del lexema kleîdi -que resuelve el enigma de la forma del caligrama. Por lo tanto, estamos en condiciones de afirmar que el escolio, respecto de su continente, es la configuración del dativo instrumental kleíde ausente en el contenido: el tekhnopaignion, una llave, es el instrumento mediante el cual es pósible acceder a una interpretación de los hexámetros 87-88 de II. Z, através de la interacción del contenido y de la forma del escolio.

El escoliasta calca sobre un juego artificioso en la distribución y en la organización de los lexemas la complejidad de la estructura que analiza, estimulando, asi, a partir de un paradigma alejandrino, la búsqueda perceptiva de claridad y de apertura en el sintagma homérico. ${ }^{(3)}$

\section{(AGRADECIMENTO)}

Deseamos expresar nuestro agradecimento a la Licenciada Elena Huber, que no sólo nos inició en el estudio de los poemas figurativos sino también nos proporcionó el material sobre el que hemos realizado el presente trabajo.

\section{ABSTRACT}

A new interpretation can be obtained confronting llias Z, 86-98 / 269-270 with an excerpt from Eustatius Comentarii ad Homeri lliadem and with a technopaegnium text. It rebuts the philological remarks made by the figurative-poem composition scholiast.

Then, the analysis of the technopaegnium takes into consideration its function and its meaning-form connection.

This work conclusion can be established from the syntactic and semantic evidence in the technopaegnium and in the lliadic hexameters.

(3) La transliteración se realizó tomando como punto de referencia la tabla propuesta por los profesores E. Prieto y R. Alcalde en su traducción de KIRK, G. S. Los Poemas de Homero. Buenos Aires: Paidós, 1962. 